\title{
From Classical Theory to Quantum Gravity
}

\author{
Bernard F. Schutz
}

Received: 6 August 2009 / Accepted: 3 September 2009 / Published online: 6 January 2010

(C) The Author(s) 2009. This article is published with open access at Springerlink.com

\begin{abstract}
While it has been generally accepted for decades that general relativity and quantum theory are inconsistent, and that therefore general relativity must yield to a new theory of gravity compatible with quantum principles, the way to the new theory is still very unclear. A major obstacle is the lack of any experimental indicators as to where general relativity might break down. I speculate on the different ways in which a quantum theory including gravity might emerge from present-day theory and use these speculations to examine a number of promising lines for new experiments.
\end{abstract}

Keywords Fundamental physics · General relativity · Tests of gravitation · Quantum gravity

\section{Introduction}

Ever since the early days of quantum theory it has been clear that general relativity and quantum theory are incompatible, and that some new theory of gravity needs to be found that will be compatible with quantum principles. However, I am aware of no proof of this, no explicit demonstration that it is impossible to marry general relativity with, say, electroweak theory, that using them together leads to a mathematical contradiction. In fact, both general relativity and our best quantum theories (take electroweak theory, for example) have internal problems: singularities evolve in general relativity from regular initial data, and there are solutions that have naked singularities that lead to acausal evolution (the Big Bang being an example); and infinite energies in standard quantum field theory can only be removed by the device of renormalization, which looks very worrying when one expects gravity to be created by all forms of energy. Moreover, quantum measurement theory as currently understood requires a distinction between the "system" and the "observer", a distinction that will be difficult to maintain if one wants a quantum formulation of the entire universe.

B.F. Schutz $(\bowtie)$

Albert Einstein Institute, Potsdam, Germany

e-mail: bernard.schutz@aei.mpg.de 
Perhaps partly because it is unclear exactly where the inconsistency between these two pillars of physics lies, there is no general agreement among theoretical physicists as to how to find the desired quantum theory of gravity. Many theorists expect that we will only have truly consistent and causal theories of gravity and matter when we find a unified quantum description of them all. String theory is today the most popular framework within which these hopes are being pursued. Others, however, look for a quantum description of spacetime itself, on the grounds that such a theory might look very different from our current paradigm about how to form a quantum theory of a matter field, and therefore one can only do the job of integrating matter fields into the picture later, once one knows what the picture looks like.

In both approaches it is generally expected that the transition from classical gravity to quantum gravity occurs at the Planck scale, variously expressed as $\ell_{\mathrm{Pl}}=\left(G \hbar / c^{3}\right)^{1 / 2}=$ $1.6 \times 10^{-33} \mathrm{~cm}, t_{\mathrm{Pl}}=\ell_{\mathrm{Pl}} / c=5 \times 10^{-44} \mathrm{~s}, m_{\mathrm{Pl}}=c^{2} \ell_{\mathrm{Pl}} / G=2 \times 10^{-5} \mathrm{~g}$, and $E_{\mathrm{Pl}}=m_{\mathrm{Pl}} c^{2}=$ $1.2 \times 10^{28} \mathrm{eV}$. This is basically a dimensional argument, so it is not difficult to devise scenarios where the transition occurs at lower energies and longer length scales. We shall meet some alternatives below. Wherever the transition might occur, presumably at smaller length scales spacetime loses its continuum character and is replaced by a probabilistic tangle of fibers, strings, loops, foam, or something even more exotic.

Although there are many ideas about how this might work, it has been remarkably difficult to find a theory or theoretical framework which remains self-consistent when developed fully. String theory is popular because it is essentially the only candidate in the spirit of a field theory that seems to be finite when all fields are included; to remain finite it needs ten dimensions. The matter fields are confined to our 4-dimensional spacetime embedded in this larger manifold, and only gravity seems to extend into the other dimensions. This picture offers rich freedom to suggest new phenomenology, depending on assumptions about the nature of the extra dimensions and the physics of the strings themselves. Particularly popular lately have been brane-world scenarios. Many string-related scenarios offer experimental access to quantum gravitational effects at scales much larger than $\ell_{\mathrm{Pl}}$.

The main alternative to string theory is loop quantum gravity, which follows the idea of quantizing gravity essentially by itself. It lives in four dimensions and has no other natural length scales, so essentially all quantum effects in loop quantum gravity occur at the Planck scale. Loop quantum gravity therefore has so far not been such a fertile source of new tests of quantum gravity. However, if it replaces continuum spacetime with a fundamentally different structure, then Lorentz invariance should be a casualty, and it is certainly at least possible that this is visible over larger distances. Moreover, the recent demonstration that, in a restricted version of loop quantum gravity, it is possible to construct a cosmological model that goes through the Big Bang without a singularity (Bojowald 2008), is exciting and offers the possibility that observations of the cosmic microwave background or of some earlier epoch might show characteristics of this process.

Experimental tests may in fact be the only way forward in the quantum-gravity program; at the very least new experimental results would be huge progress. The problem is daunting if one believes that new results will only be found on the Planck scale. The highest energies accessible to us at present are from ultra-high-energy cosmic rays, the highest of whose energies approach $10^{22} \mathrm{eV}$. (See the contribution from Watson in this volume.) But it is possible that at low energies there are some residual effects of quantization that are still visible, or that some of the extra dimensions in string theory are larger than the Planck length, which would make new experimental tests more accessible. Another type of theory, emergent gravity, offers the same possibility. I will discuss all these possibilities below. 


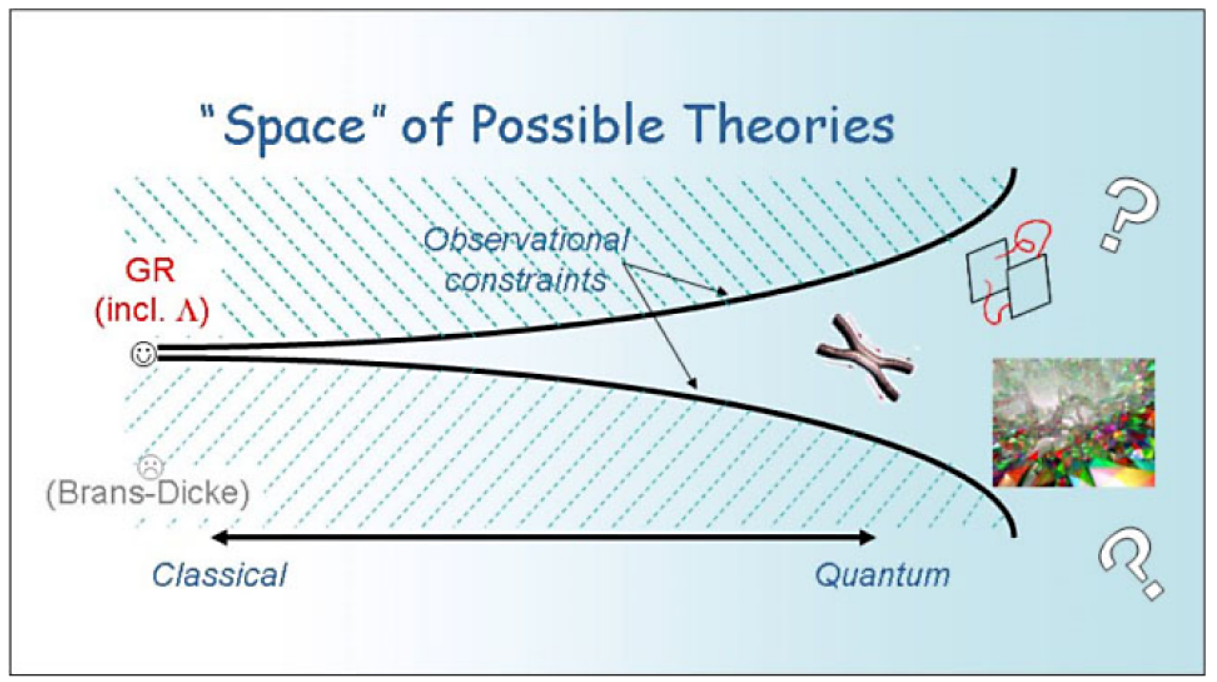

Fig. 1 Experimental constraints at low energies point very strongly to general relativity with a cosmological constant, excluding for example Brans-Dicke Theory for small values of the coupling parameter. At high energies, as one approaches the quantum regime, the allowed space of theories widens and is constrained more by mathematical consistency than by experiment

The central problem for the experimental search for quantum gravity is that, so far, there are no observed deviations of general relativity from experiment. Unlike the situation at the beginning of quantum theory, where there was a host of unexplained spectroscopic evidence and some very important particle-scattering experiments, today we have nothing to try to explain or fit. General relativity with a cosmological constant $(\Lambda \mathrm{GR})$ is all we need at present. (See the contribution from Clifford Will in this volume.) The situation is illustrated in Fig. 1: constraints on gravitation theories at low energies are very strong, but as we approach the quantum scale these same constraints provide less and less guidance.

There is, of course, some room for worry. The cosmological constant is very small compared to the "natural" or non-renormalized vacuum energy density that quantum field theory would suggest, so its non-zero value might be a strong clue to the integration of quantum theory and gravity; however, no-one yet has worked out how to use that clue successfully. Alternatively, the dark energy might not be a cosmological constant; it might evolve with time in a way that depends on the energy density or expansion rate of the universe. That would also be a clue, at least to a new cosmological field that might be a product of the marrying of gravity and quantum theory; here the problem is that theorists have proposed a wealth of such "quintessence" fields, and there is not enough precision in the cosmological observations so far to pin down which, if any, is right. One could also take the evidence for dark matter as evidence instead for modified gravity. Since we have not yet identified the dark matter particle, perhaps some other theory that modifies gravity at large length scales (Milgrom 2008; Bekenstein 2004) is better than general relativity, and then its extra fields would be explained as low-energy effects of a quantum gravity theory. All of these alternatives will be discussed in this volume.

The rest of this paper is organized into two main parts. The first reviews the different approaches to extending general relativity into a quantum theory and what observable differences they might produce. The second part then suggests what kinds of experiments might provide insight into the path beyond general relativity. 


\section{How to Break General Relativity}

\subsection{Low-energy Effects of Quantum Gravity}

Planck-scale changes in gravity may themselves have residual effects at low energies and long length scales. This could happen in a number of ways.

First, the full quantum theory itself might have unusual features that persist at low energies. Supersymmetry is one: superstring theory is the preferred variant at present because supersymmetry leads to cancellations between the zero-point energies of the normal particles and their supersymmetric counterparts (sparticles), leading naturally to a zero or at least a small cosmological constant. The discovery of a sparticle when the LHC reaches its full operating energy at CERN would greatly narrow down the space of possible approaches to quantum gravity.

Second, there could be fields that arise from the full theory but which are still present and detectable at low energies today. Many low-energy effective theories introduce massless scalar fields that couple, say, to baryon number and therefore behave very like a scalar gravitational field. Radiation of such a field would carry energy away from a gravitational wave source, altering its long-term evolution and waveform in potentially detectable ways, while the radiation itself could be distinguished from standard tensor gravitational waves by its distinctive polarization pattern. It might happen that the full theory has an effective Lagrangian that has a Chern-Simons-type term, breaking parity invariance. This would have distinctly observable effects in gravitational waves from binary systems: see Alexander and Yunes (2008).

It is also possible that, if the full theory includes grand unification at very high energies, then spontaneous symmetry breaking during the expansion of the universe could have produced, besides standard matter, a completely separate matter sector that has its own internal interactions (analogous to our strong, weak, and electromagnetic interactions) but whose only coupling to standard matter is through gravity. Such "shadow matter" would be detectable by gravitational means but not otherwise.

Third, there could be fossils of high-energy systems that still live on. For example, Damour and Vilenkin (2005) have suggested that fundamental strings, which are the building blocks of particles in string theory, could be turned into macroscopic cosmic strings by inflation, in the same way that inflation turns quantum fluctuations into the real density fluctuations that are measured in the cosmic microwave background. These cosmic strings would emit gravitational waves with a distinctive signature, and their detection would be a big step toward verifying the string theory framework.

Low-energy effects can also be searched for by performing highly sensitive null experiments, such as looking for temporal variations in the fine-structure constant, for violations of the local isotropy of space, or for violations of local Lorentz invariance. While we are unlikely to measure Planck-level corrections to $g_{00}$ any time soon, an extra field introduced by quantum effects might be visible where general relativity predicts a zero.

A special kind of null experiment is the nulling of fringes in an interferometer. Precisely here there has recently been a suggestion from Hogan (2008) that quantum spacetime effects could produce a fundamental noise with a spectral noise density $S_{h} \sim t_{\mathrm{Pl}}$. It turns out that modern gravitational wave interferometers operate near this level, and in fact that the GEO600 interferometer has for years had an unexplained noise of this size. Hogan derives his effect from a holographic approach to spacetime information theory; as such it does not fit into standard quantum gravity schemes. If this noise could be proved to exist, it would revolutionize the search for quantum gravity. 
Finally, it is well to recall that in at least one area we are only about 6 orders of magnitude away from the Planck scale. Ultra-high-energy cosmic rays (UHCRs) have been measured at energies approaching $10^{22} \mathrm{eV}$. If these consist of single particles, then their individual energies are as large as $10^{-6} E_{\mathrm{Pl}}$. If there is a violation of Lorentz invariance at Planck energies, which seems very possible, then ultra-precise observations of these particles (hard to do!) might reveal it. Of course, when they interact with atmospheric hadrons the centerof-mass energies are much smaller, of order $10^{15} \mathrm{eV}$. This is still above the energy of the LHC, so supersymmetry and other effects might be manifest in the collisions of UHCRs with atmospheric nuclei, but the energies are probably too far from Planck for direct effects of quantum gravity to be visible.

\subsection{Models Involving Extra Dimensions}

Our universe is a 4-dimensional subspace of the 10- or 11-dimensional space in which string theory is finite. In the most conservative picture, the extra dimensions are all curled up, as in Kaluza-Klein models, on a Planck length-scale. But larger dimensions are possible. A lot of attention has been paid recently to brane-world models, where we live on a 4-brane and there could be other branes embedded in this larger space. It is possible that one or more dimensions is larger, and either wraps up over a longer length scale or terminates on another brane some distance away. In such a picture, the conventional three interactions of particle physics would be confined to our brane, but gravity would extend into the other dimensions (called the bulk) as well. To be consistent with current limits on the Newtonian inverse-square-law, the size of these "large" dimensions would need to be smaller than a millimeter or so. Nevertheless, this would be 32 orders of magnitude longer than $\ell_{\mathrm{Pl}}$, and would potentially bring new effects into physics at energies as low as $10^{-4} \mathrm{eV}$.

The most direct test of large extra dimensions is to measure the gravitational inversesquare law at sub-millimeter lengths, a big challenge. (See the contribution of Gundlach to this volume.) The gravitational acceleration produced by a point mass in $n$ dimensions falls off as $r^{-(n-1)}$ (for $n>1$ ), so if below some length-scale gravity could sense the extra dimensions, then it would get strong faster at smaller distances than one expects in three dimensions.

Other tests are possible, however. There have been plenty of novel predictions and speculations. A number of scenarios within the brane picture are reviewed by Maartens (2004). For example, branes could collide, even giving rise to the Big Bang itself, or creating gravitational waves. Nearby branes could influence our own brane gravitationally, so that we might detect gravitational waves radiated by a binary system in a parallel universe!

\subsection{Alternative Approaches}

Although most work on quantum gravity today is being done within the string theory and loop quantum gravity frameworks, there are alternatives, and each makes distinct predictions.

There is a program to derive continuum spacetime as a limit of discrete causal sets due to Sorkin (2007). This approach may make predictions about a variety of testable violations, such as Lorentz invariance and the time-behavior of the dark energy.

Penrose (2002) has argued that measurement theory will be affected by gravity, and in particular that the distinction between the observer and the system will come about in a natural way by decoherence of the wave-function induced by gravity. If this could be shown experimentally, it would have a very strong influence on how quantum gravity progressed. 
General relativity might not be the fundamental field we think it is. Instead it could be an emergent phenomenon, analogous to the way that superfluidity emerges from detailed molecular interactions: see Volovik (2003). In this case, the Planck scale is an illusion created by the weakness of gravity, whereas the fundamental physics is happening at other energy scales, perhaps much lower than $E_{\mathrm{Pl}}$. I am not aware of concrete predictions from this point of view, but certainly any anomalous experimental result showing a violation of general relativity could point in this direction.

\subsection{Dark Energy}

The cosmological constant $\Lambda$ represents a field whose energy density $\rho$ is invariant under Lorentz transformation. In order to have this property, it must necessarily have negative pressure $p=-\rho$. It arises naturally in quantum field theories, since any zero-point energy density of a relativistic field must be Lorentz invariant. But typically these are large and have to be renormalized away. Supersymmetry does this automatically, perhaps too efficiently, since it might be hard to get any non-zero $\Lambda$ in the end.

Theorists have therefore invented other physical fields that have almost but not quite invariant energy densities. Generally these are called quintessence theories. They can have an equation of state (relation of $p$ and $\rho$ ) that changes with time. Precision cosmological observations are necessary in order to distinguish these from a cosmological constant and from one another.

Emergent theories also lead to a cosmological constant, but not to a particularly small one.

\section{Where to Look for Broken General Relativity}

After this survey of the theoretical perspectives on quantum gravity, a number of experimental directions seem natural.

Cosmological observations with high precision are very important, and should be done out to redshifts up to 2 or beyond. If it turns out that the dark energy really is a cosmological constant, then that kicks the ball back to conventional theories. But if there is some kind of evolution in the dark energy, that could be a real clue. Relevant space missions include JDEM and LISA. Ground-based observing programs can also make decisive contributions.

Observations of a cosmological background of gravitational waves would also give strong clues: the waves might arise from inflation, but stronger backgrounds could come from symmetry breaking as the universe expands, from brane-world effects, from shadow matter, and so on. Here one looks to the gravitational wave detectors LIGO (Abbott 2009), VIRGO (Acernese et al. 2008), and LISA, and even more to the next-generation detector now being called the Einstein Telescope (ET).

There may be clues in gravitational wave propagation effects. If the graviton has some kind of effective mass, this could lead to dispersion and anomalous time-delays within signals, measurable by LISA, LIGO, VIRGO, or ET. A Chern-Simons term, mentioned earlier, could make the two components of circular polarization propagate at different speeds, leading to observable effects in observations of signals from binary systems detected by LISA (Alexander and Yunes 2008).

Experiments that look for violations of local Lorentz invariance and other principles of local physics are potentially very informative; they are null experiments and so even when they produce no observable violation they produce interesting constraints on the terms in a 
theory that lead to violations. The equivalence principle is expected to be violated at some level; astonishingly accurate laboratory experiments (Schlamminger et al. 2008) will soon be superseded by space missions like MICROSCOPE (Touboul et al. 2006), GG (Nobili et al. 2009), and possibly STEP (Overduin et al. 2009) that could push the limits to parts in $10^{18}$. Temporal or spatial variations in the fundamental constants would give evidence of how spontaneous symmetry breaking happens, or of unexpected couplings between fields, or of incomplete homogenization by inflation. Temporal variations can be explored in local experiments, and there are several interesting proposed space missions. Spatial variations presumably happen only over cosmological distances, and to detect them requires very precise spectroscopic observations of distant sources like quasars. Space could be anisotropic even locally, which would be evidence of new tensor fields. And, perhaps most interestingly, Lorentz invariance could fail. If this happens on macroscopic scales, it might indicate preferred frames or new long-range forces; if it happens in tiny domains then this might indicate the length-scale of the transition to quantum gravity.

Testing general relativity itself has been an important activity ever since the first observations of the deflection of light by the Sun. Solar-system tests in the PPN framework have so far not revealed any difficulties with general relativity, but they are only just beginning to probe second post-Newtonian effects. Radio observations of pulsars in binaries, and particularly of the double pulsar system PSRJ 0737-3039, test the radiative part of general relativity, are becoming accurate enough also to test higher-order post-Newtonian effects on the orbital motions, and are also able to probe wider aspects of gravity, such as preferred-frame effects Lorimer (2008), Stairs (2003). Observations of binary inspiral using gravitational wave detectors will someday, at the latest when LISA flies, provide strong constraints on post-Newtonian gravity. Such observations can also test other aspects of general relativity, such as whether waves have just two transverse tensorial polarization states, whether there is frequency dispersion of gravitational waves, and whether right- and left-handed circularly polarized waves travel at the same speed. LISA and ET will also make observations of binary black hole mergers with sufficient precision to place good constraints on the black hole uniqueness theorem, the Hawking area theorem, and the cosmic censorship conjecture.

As we have seen, testing Newtonian gravity at short range could reveal the length scale where quantum effects set in, if large-size extra dimensions play a role (Gundlach et al. 2009 , this issue). Newtonian gravity over long ranges could also show up quantum effects. Some or all of the anomalous gravitational effects attributed to dark matter could in principle be an indication of the violation of general relativity over long distances.

Finally, experimenting on quantum physics over large distance scales might possibly reveal anomalies. If gravity plays a role in decoherence then this could be testable in space. There are interesting mission proposals for experiments on entangled states over very large distances that could shed light on how standard quantum measurement theory should be extended to quantum gravity. This area has had less theoretical attention than quantization itself has (Penrose 2002).

\section{Conclusions}

While it is plausible that pointers to quantum gravity exist at low energies, there is no guarantee, and so one should be cautious before deciding to invest in a delicate and possibly expensive experiment. We have no clues so far to the strength of violations of general relativity: theories have too many adjustable parameters. It could happen that general relativity is "clean" all the way down to the Planck scale! 
That would, however, be very surprising in light of the complexity of physics at energies accessible to us. If some form of grand unification holds, then it would be remarkable if we already see all the physics there is, and nothing new emerges right up to the unification energy that could have effects at our energies. So the motivation to find new effects is strong, and the difficulty is to know which direction to look.

Fortunately, some of the ways of investigating these problems are by-products of experiments and observation programs that have other motivations. Cosmological observations of the dark energy and gravitational wave observations fit into these categories. So do precise spectroscopic observations of quasars. Any of these could turn up the first evidence and point the way to more dedicated experiments in fundamental physics.

Alongside these, there is strong motivation to do laboratory experiments to look for violations of our standard expectations, such as the equivalence principle or the inverse-square force. These are constant sources of technological innovation. And as null experiments, even a null result is a useful constraint on theory.

In the last decade a number of space missions have been proposed to test general relativity and look for quantum effects. GP-B (Silbergleit et al. 2009, this issue) and the LAGEOS (Ciufolini 2007) observations measure a non-zero effect, the gravitomagnetic part of general relativity. MICROSCOPE will be the first null experiment for gravity in space. The European Space Agency's call for mission proposals in the Horizons 2000+ program produced a set of very interesting proposals using cold atom technology to do null experiments and to study quantum measurement theory. While none were selected for study in the first round, funding is being made available for technology development; there is a strong motivation to do so, not just because of the insight these experiments will give into quantum gravity, but because of the potential further applications of these new technologies.

Ultimately, despite the uncertainty about what the right direction might be for an experiment, the motivation for doing the experiments is that finding a quantum theory of gravitation is the single most important outstanding problem in our understanding of fundamental physics today. And the search for this quantum theory is starved of experimental data to guide it.

Every experiment is a step in the right direction.

Open Access This article is distributed under the terms of the Creative Commons Attribution Noncommercial License which permits any noncommercial use, distribution, and reproduction in any medium, provided the original author(s) and source are credited.

\section{References}

B.P. Abbott (The LIGO Scientific Collaboration), LIGO: the laser interferometer gravitational-wave observatory. Rep. Progr. Phys. 72(7), 076901 (2009)

F. Acernese, M. Alshourbagy, P. Amico, F. Antonucci, S. Aoudia, P. Astone, S. Avino, L. Baggio, F. Barone, L. Barsotti, M. Barsuglia, T.S. Bauer, S. Bigotta, S. Birindelli, M. Bizouard, C. Boccara, F. Bondu, L. Bosi, S. Braccini, C. Bradaschia, A. Brillet, V. Brisson, D. Buskulic, G. Cagnoli, E. Calloni, E. Campagna, F. Cavalier, R. Cavalieri, G. Cella, E. Cesarini, E. C-Mottin, A.-C. Clapson, F. Cleva, E. Coccia, C. Corda, A. Corsi, F. Cottone, J.-P. Coulon, E. Cuoco, S. D’Antonio, A. Dari, V. Dattilo, M. Davier, R. DeRosa, M. DelPrete, L. Di Fiore, A. Di Lieto, A. Di Virgilio, B. Dujardin, M. Evans, V. Fafone, I. Ferrante, F. Fidecaro, I. Fiori, R. Flaminio, J.-D. Fournier, S. Frasca, F. Frasconi, L. Gammaitoni, F. Garufi, E. Genin, A. Gennai, A. Giazotto, L. Giordano, V. Granata, C. Greverie, D. Grosjean, G. Guidi, S. Hamdani, S. Hebri, H. Heitmann, P. Hello, D. Huet, S. Kreckelbergh, P. La Penna, M. Laval, N. Leroy, N. Letendre, B. Lopez, M. Lorenzini, V. Loriette, G. Losurdo, J.-M. Mackowski, E. Majorana, M. Mantovani, F. Marchesoni, F. Marion, J. Marque, F. Martelli, A. Masserot, F. Menzinger, L. Milano, Y. Minenkov, C. Moins, J. Moreau, N. Morgado, S. Mosca, B. Mours, C.N. Man, I. Neri, F. Nocera, 
G. Pagliaroli, C. Palomba, F. Paoletti, S. Pardi, A. Pasqualetti, R. Passaquieti, D. Passuello, F. Piergiovanni, L. Pinard, R. Poggiani, M. Punturo, P. Puppo, P. Rapagnani, T. Regimbau, A. Remillieux, F. Ricci, I. Ricciardi, A. Rocchi, L. Rolland, R. Romano, P. Ruggi, G. Russo, S. Solimeno, A. Spallicci, M. Tarallo, R. Terenzi, A. Toncelli, M. Tonelli, E. Tournefier, F. Travasso, C. Tremola, G. Vajente, J.F.J. van der Brand, S. van der Putten, D. Verkindt, F. Vetrano, A. Vicerè, J.-Y. Vinet, H. Vocca, M. Yvert, VIRGO: a large interferometer for gravitational wave detection started its first scientific run. J. Phys. Conf. Ser. 120(3), 032007 (2008)

S. Alexander, N. Yunes, Chern-Simons modified general relativity. Phys. Rev. D 77, 124040 (2008)

J.D. Bekenstein, Relativistic gravitation theory for the modified Newtonian dynamics paradigm. Phys. Rev. D 70(8), 083509 (2004)

M. Bojowald, Loop quantum cosmology. Living Rev. Relativ. 11(4) (2008)

I. Ciufolini, Dragging of inertial frames. Living Rev. Relativ. 449, 41-47 (2007)

T. Damour, A. Vilenkin, Gravitational radiation from cosmic (super)strings: bursts, stochastic background, and observational windows. Phys. Rev. D 71, 063510 (2005)

J.H. Gundlach, S. Schlamminger, T. Wagner, Laboratory tests of the equivalence principle at the University of Washington. Space Sci. Rev. (2009). doi:10.1007/s11214-009-9609-3

C.J. Hogan, Indeterminacy of holographic quantum geometry. Phys. Rev. D 78(8), 087501 (2008)

D.R. Lorimer, Binary and millisecond pulsars. Living Rev. Relativ. 11(8) (2008)

R. Maartens, Brane-world gravity. Living Rev. Relativ. 7, 7 (2004)

M. Milgrom, The MOND paradigm. arXiv:0801.3133v2 (2008)

A.M. Nobili, G.L. Comandi, S. Doravari, D. Bramanti, R. Kumar, F. Maccarrone, E. Polacco, S.G. Turyshev, M. Shao, J. Lipa, H. Dittus, C. Laemmerzhal, A. Peters, J. Mueller, C.S. Unnikrishnan, I.W. Roxburgh, A. Brillet, C. Marchal, J. Luo, J. van der Ha, V. Milyukov, V. Iafolla, D. Lucchesi, P. Tortora, P. de Bernardis, F. Palmonari, S. Focardi, D. Zanello, S. Monaco, G. Mengali, L. Anselmo, L. Iorio, Z. Knezevic, "Galileo Galilei" (GG) a small satellite to test the equivalence principle of Galileo, Newton and Einstein. Exp. Astron. 23, 689-710 (2009)

J. Overduin, F. Everitt, J. Mester, P. Worden, The science case for STEP. Adv. Space Res. 43, 1532-1537 (2009)

R. Penrose, Gravitational collapse of the wavefunction: an experimentally testable proposal, in The Ninth Marcel Grossmann Meeting, ed. by V.G. Gurzadyan, R.T. Jantzen, R. Ruffini (2002), pp. 3-6

S. Schlamminger, K.-Y. Choi, T.A. Wagner, J.H. Gundlach, E.G. Adelberger, Test of the equivalence principle using a rotating torsion balance. Phys. Rev. Lett. 100(4), 041101 (2008)

A. Silbergleit, J. Conklin, D. Debra, M. Dolphin, G. Keiser, J. Kozaczuk, D. Santiago, M. Salomon, P. Worden, Polhode motion, trapped flux, and the GP-B science data analysis. Space Sci. Rev. (2009). doi:10.1007/s11214-009-9548-Z

R.D. Sorkin, Is the cosmological "constant" a nonlocal quantum residue of discreteness of the causal set type? in Particles, Strings, and Cosmology-PASCOS 2007, ed. by A. Rajantie, C. Contaldi, P. Dauncey, H. Stoica. American Institute of Physics Conference Series, vol. 957 (AIP, New York, 2007), pp. 142153

I.H. Stairs, Testing general relativity with pulsar timing. Living Rev. Relativ. 6(5) (2003)

P. Touboul, R. Chhun, M. Rodrigues, B. Foulon, E. Guiu, MICROSCOPE, equivalence principle test with a micro-satellite, in 36th COSPAR Scientific Assembly. COSPAR, Plenary Meeting, vol. 36 (2006), p. 3585

G.E. Volovik, The Universe in a Helium Droplet (Oxford University Press, Oxford, 2003) 